\title{
Analysis of the Romanian National Data System on Child Abuse and Neglect
}

\author{
Gabriella Tonk ${ }^{1}$, Júlia Szigeti ${ }^{*}$ \\ ${ }^{1}$ Babeș-Bolyai University of Cluj-Napoca, Department of Social Work, 128-130 21 Decembrie 1989 Bld., 400604 Cluj-Napoca, \\ Romania
}

KEYWORDS

Abuse

Child

Data system

Database

Neglect

\section{A BSTRACT}

This article proposes to perform a mainly qualitative analysis of the national data collection system on child abuse and neglect from the perspective of the right of the child to protection, participation and development.

The study performed with the initiative of ChildONEurope network identifies a few characteristics of good practice regarding the development of complex databases on child abuse and neglect. These characteristics are organized around three elements that will constitute analyzing criteria for the present study, applied to the Romanian data systems. The three elements are: objectives, criteria and resources.

The main scopes of the study are: 1 . Presenting the Romanian data system regarding child abuse and neglect; 2. Identifying the strengths and weaknesses of the Romanian data system based on the above mentioned criteria; 3. Identifying new directions for development of the data collection and monitoring the Romanian child protection system.

In order to achieve the above mentioned scopes, a qualitative analysis has been made of the Romanian institutional-legislative framework, through document analysis, completed with an interview with the representative of the National Authority for Protection of Family and Child's Rights. Here the study aims to identify the possible gaps and ambiguousness of the legal framework that could be one of the sources of unreliable data.

The analysis of the national database has been made through document analysis, while one of county level databases has been made through implementation of a questionnaire in 47 county directorates for social assistance and child protection, completed with 3 interviews with representatives of two local institutions from Cluj County.

\footnotetext{
*Contact addresses: gszabo2002@yahoo.com (G. Tonk), juliaszigeti@yahoo.com (J. Szigeti)
} 


\section{Introduction}

The official statistics regarding the number of children who suffered from abuse and neglect represents only the top of the iceberg, which contains cases registered by the authorities. Definitions, classifications, identifying and evaluation procedures has major impact on what do official statistics reveal in a country.

In Romania, similarly to other countries, there is a huge discrepancy between the number of identified child abuse and neglect cases, registered in the national database and the prevalence of abuse and neglect, highlighted by the few studies from the country.

This paper proposes to perform a mainly qualitative analysis of the national data collection system on child abuse and neglect from the perspective of the right of the child to protection, participation and development. The analysis of the national and local databases has the aim to identify new directions for development of the data collection and monitoring of the Romanian child protection system.

\section{Theoretical framework}

The child's rights perspective in the analysis of state response to the issue of child maltreatment

Frequently, the issue of child maltreatment is considered to be a public health issue whith devastating effects upon individuals, families and communities. It is more rarely considered to be a human rights issue.

Researching child abuse and neglect using the public health approach usually covers the topic of monitoring, identifying and diagnosing cases, investment in early prevention, cost-efficiency, access to support and recovery services.

The child protection approach analyzes the issue of child maltreatment from the perspective of the taken professional and legal actions.

At the same time the children's right approach is necessary to be used, defining child maltreatment as a human right violation involving the 
responsibility of the state to act, in accordance to widely accepted international treaties. (Reading et al. 2009) The great advantage of children's right approach, based on the UN Convention of the Rights of the Child is the assurance of legal instrument for implementation of policies, responsibilities and social justice. Child maltreatment must be considered a public health issue, as well as a human right one.

The specific data on child maltreatment are crucial to determine the extent of state's ability to observe its responsibility to protect children from violence, to develop the protection and prevention system and direct resources to the domains that are in greatest need.

The need of valid and accurate data is stressed by many programmatic documents of UN and European institutions - e.g. Decision of European Parliament and Council for the institution of a second phase of a Daphne Program, 21 April 2004, WHO 2002, Report on Health and violence, World Fit for Children (2002) - which ask for development of national data systems on child abuse and neglect. The same recommendation is made by the UN Committee on the Rights of the Child towards the Romanian third and fourth submitted report, namely to develop a nationwide data collection and analysis system in order to produce comparable and complementary data regarding all issues of children's rights (UN Committee on the Rights of the Child).

Collection, analysis and dissemination of data regarding child maltreatment serve the health and physical, emotional, social and cognitive well-being of children and youth (Fluke et al. 2009).

Within the statement of priorities, program projecting and progress monitoring it is vital that the capacity of data collection and analysis be increased.

The importance of analysis of collected data is stressed by Finkhelor (2001) and his collaborators who tried to find explanations to the fact that is the United States the number of substantiated sexual abuse cases decreased with 39\% between 1992 and 1999, according to National Child Abuse and Neglect Data System. The study draws attention to the fact that this descending trend wasn't analyzed. According to the researchers the descent might indicate a real reduction of sexual abuse cases but could also mark the decrease of the registered cases, without any change of prevalence, 
mentality, politics and standards. Conclusions show that there is a great need for improvement of databases, for allocation of more resources to the analysis and utilization of data and for training of professionals regarding the importance of using data in the process of policy development.

In Romania very few national studies were conducted, aiming to map the prevalence of child abuse and neglect. In this regard, the research made by Save the Children Romania (Alexandrescu 2000) revealed that $47 \%$ of the parents declared that they use violence as a method for discipline, while $84 \%$ of the children declared that they were subjected to violent actions from their parents. $20 \%$ of these children reported that they were beaten with objects and 15\% declared that they are afraid to go home because of violence.

The national statistics shows the incidence of abuse and neglect, but many researches state the fact that the majority of victims did not disclose the abuse ever (Gough and Lynch 2000; Creighton and Russell 1995; Palmer et al. 1999).

The cause of underreporting is related partly with the characteristics of the phenomena of abuse and neglect - e.g. sexual abuse occurred in places with no witnesses, victims are afraid of blaming or revenge - partly with some cultural dynamics that accepts some forms of physical and emotional abuse as forms of discipline. Other forms are not recognized as such, if the child is not a direct victim (the case of domestic violence).

On the other hand, studies show a great tolerance and lack of interest of the community regarding the issue of child maltreatment. Here we can add the unclear legislation regarding responsibilities, lack of an appropriate evaluation, of communication between agencies and departments and of the proper working instruments.

Studies (European Network of National Observatories on Childhood) show that there are some causes for the lack of interest displayed by institutions, professionals and policy makers towards their own data regarding child maltreatment, such as lack of trust in their own data system, lack of resource allocation for data interpretation, lack of orientation towards a systematic evaluation of performance, fear to publish data, fear of resource reduction in case if the phenomenon declines. 
The study of data collection methods involves the observance of the variations of the abuse and neglect definitions that are used by different sectors related to the issue. At the same time, the problem of conceptualization arises (because abuse and neglect have different aspects such as clinical, legal, social and administrative), as well as the problem of operationalization (which actions are considered to be abusive) and that of registration (which cases are registered as such: all reported cases or just substantiated cases). These are just a few questions that need to be answered in order to have a uniform and integrated data system.

Due to the complexity of the phenomenon studies show that it is difficult for states to integrate data resulting from the justice and social system, without common definitions and integrating mechanisms. Studies also suggest that social protection and health care systems are most suitable for data collection considering the long-term consequences of abuse. At the same time, social and health services are the most important interfaces to identify and reveal abuse, if screening methods are used, due to the fact that these institutions have contact with a large number of families, looking for services for other needs than violence. Data resultant from social services helps a lot in estimation of the phenomenon and identifying its characteristics.

Administrative data regarding reported, investigated, and substantiated cases consequently have a great deal of importance, keeping in mind however their limitations, in identifying national trends and transnational comparisons too (European Network of National Observatories on Childhood).

The study initiated by the European Network of National Observatories on Childhood regarding institutional framework of data collection and national data systems on child abuse and neglect has been conducted in 20 European countries (Romania was not among them) showed interesting results:

The deterioration of health sector as data source for child abuse and neglect has been determined.

- Though almost all countries introduced in their legislation the obligation of reporting for the professionals, this has not necessarily lead to an improvement of data collection. 
As a consequence of the great variation of uncoordinated data sources the analysis of abuse rarely has the child in the center and information like child's situation and characteristics of abuse is rarely integrated with information regarding taken measures.

- Within a child-centered data system an identification code for each child must used in order to avoid duplication and to monitor the evolution of the case in the child protection system. Codification should follow the registration and monitoring methods used in health care system.

- Obviously, the direction of transmitting data is from local to central. Good practice constitutes the development of guidelines for reporting and register cases. A middle-level (e.g. regional) integration and control of data is recommended.

- Structural differences in data collection methods existing between social, judicial and health sectors are hard to overcome, but establishment of a minimum common set of registered variables could make possible the comparison of cases reported to different sectors. These, such as age, sex, relation with the perpetrator, family status etc. could be added to the sector specific variables.

- It is important to use electronic databases that ensure anonymity and confidentiality for the cases.

- There is a need to allocate resources for infrastructure, training, administration, monitoring, analysis and reporting.

On the other hand, the study shows that the variables that must be registered and monitored are the risk and protective factors identified by numerous studies and the interventions and actions that has been taken is the respective case. A complete representation of the phenomena must have data regarding socio-demographic characteristics of the child victim, sociodemographic characteristics of the perpetrator, intervention, professionals and services involved, duration of intervention and costs. This information can constitute the bases for primary, secondary and tertiary prevention, for establishing risk and protective factors, incidence, evaluation of interventions and monitoring the efficiency of the policies. 


\section{Criteria for analysis of Romanian data system on child abuse and neglect}

The study conducted by the European Network of National Observatories on Childhood identifies a few characteristics of good practice regarding the development of complex databases on child abuse and neglect. These characteristics are organized around three elements that will constitute analyzing criteria for the present study, applied to the Romanian data systems.

The first element is the objective that is served by the database. The most relevant objectives identified by the European Network of National Observatories on Childhood are the following: monitoring of the phenomenon; data collection in order to evaluate interventions and policies; administrative control and planning of personal, instrumental and financial allocation; improvement of cohesion between policy and practice at central and local level in child protection.

The following relevant characteristics were identified: clear definitions and procedures; identification of responsible institutions on central, regional and local level; clear methodologies for professionals from local levels, set of descriptive variables regarding the situation, measures and context; involvement of professionals in data analysis and discussing results; data collection and periodical reporting in order to give the chance to professionals to check their own work through statistical data.

The availability of the following resources are considered important: protocols which coordinates the different data generating sectors; standardized forms for reporting and registration; guidelines for implementation of definitions and methodologies; electronic database and user-friendly software for archive; training for improvement and implementation of the system; obligation of reporting and sanctions in case of lack of observance of reporting duty; financial allocation for updating and development of the system. 


\section{Methods}

The main scopes of the study are: 1 . presenting the Romanian data system regarding child abuse and neglect; 2 . identifying the strengths and weaknesses of the Romanian data system based on the above mentioned criteria; 3. identifying new directions for development of the data collection and monitoring the Romanian child protection system.

In order to achieve the above mentioned scopes, we made the following steps: a qualitative analysis has been made of the Romanian institutional-legislative framework, regarding data collection and monitoring of children's rights at central and local level, through document analysis, completed with an interview with the representative of the National Authority for Protection of Family and Child's Rights. Here the study aims to identify the possible gaps and ambiguousness of the legal framework that could be one of the sources of unreliable data.

The analysis of the national database has been made through document analysis, while one of county level databases has been made through implementation of a questionnaire in 47 county directorates for social assistance and child protection. This was completed with 3 interviews with representatives of two local institutions from Cluj County: one social worker from the abuse department and one social worker from the monitoring department of the county directorate for social assistance and child protection, respectively the head of child protection department of the public social service of the Municipality of Cluj.

The base for analysis constitutes the criteria described above that were considered in every level of the analysis, depending on their relevance in the Romanian system.

The questionnaire we used is one developed in the project Balcan Epidemiological Study on Child Abuse and Neglect, completed with 10 more questions, relevant for our study. It contains 6 sets of questions as the following: general information of data registering regarding child abuse and neglect; accessibility of data; monitoring report data regarding the victim; Available data regarding the incidence; Available data regarding family; Available data regarding perpetrator. The questionnaire was pretested with four institutions, after few modifications was sent to the National Authority for Protection of Family and Child's Rights which sent to the 47 county 74 
directorates for social assistance and child protection, out of which 45 has filled in and sent back the questionnaire. It has been filled in by a representative of the county directorates, from the specialized service for abuse and neglect.

The interview was used as a complementary method to complete the information obtained from the questionnaire. There have been conducted 3 semi-structured interviews in Cluj County, 2 with the representative of the county directorate's abuse service and 1 with the representative of the Public Social Service of Cluj Napoca Municipality.

The interview guide contained 5 topics: case identification; relationship between county and municipal social services; characteristics of data and monitoring.

One interview has been made with the representative of the National Authority for Protection of Family and Child's Rights regarding the national database, in order to complete the information obtained from document analysis.

\section{Analysis and results}

The Romanian data system on child abuse and neglect

Due to the fact that Romania has a centrally organized child's rights monitoring system and the responsibility of implementation of child protection policies is decentralized, the central data system differs from the local one. The present paper envisages both levels, which will be presented separately.

On central level, according to Law 272/2004 for protection and promotion of the rights of the child, until June 2010, the National Authority for Protection of Family and Child's Rights was the responsible institution for policy making and monitoring in child protection issues. Government Ordinance no. 68/2010 enacts the reorganization of these institutions inside of the Ministry of Labor, Family and Social Protection, as a specialized department. Romania has a national statistical system for monitoring the observance of children's rights since 2007, but the systematic data collection has started in 2004. 
The monitoring instrument is the Quarterly monitoring report, based on provisions of the UN Convention on the Rights of the Child.

The data collected regarding child maltreatment is concerned to children who are or were in contact with the child protection services needing secondary or tertiary prevention measures.

\section{The scope of monitoring and data validity}

Based on the interview with the representative of the National Authority for Protection of Family and Child's Rights turned out that the central authority is not satisfied with the results of implementation of the monitoring report. In spite of the fact that the main scope of implementing the instrument was mapping the phenomenon of abuse and neglect in order to support policies, decision making and programming, presently the monitoring report only reflects the development level of county directorate concerning reporting capacity.

The implementation of the monitoring report had less benefit than we expected (...) due to the personnel fluctuation, people hardly understood the indicators of the monitoring report and the way it should be fill out. I have doubts regarding the accuracy of reported cases. We only work with county directorates but we all know that on local [commune and municipal] level there are more unidentified cases. The problem remains the same: as long as the local public services are not sufficiently involved, we'll never have a real picture of the phenomenon. (representative of NAPFCR).

Another question regarding data validity is raised by the reason of the court or administrative decision which separates the child from his family. Table no. 1 shows that the rate of abuse and neglect indicated as the reason of separation is only $21.69 \%$ compared to poverty, which is the cause in $44.27 \%$ of the cases.

This could mean on one hand that children are separated from their families without a legal base, Law 272/2004 stipulates that separation of the child must be prevented even with financial support of the family, on the other hand we don't have the certitude that a valid evaluation of abuse experience was made in case of all children:

According to the legislation children can be separated from their families only in case of abuse, neglect, exploitation, theoretically. We know that the 
practice is different; children enter to the protection system due to poverty as well. It is clear that there are associated factors also, it is very hard to draw a line between poverty and neglect (...). The instructions for filling the monitoring report specify that the most relevant reason must be considered when it comes to the cause of separation (representative of NAPFCR).

Table 1. Causes of separation of children from their families who have special protection measure according to Law 272/2004, at 31.12.2009 (official statistics, www.copii.ro)

\begin{tabular}{|l|l|}
\hline Cause of separation & $\begin{array}{l}\text { Percent out of the total } \\
\text { number of children from the } \\
\text { special protection system }\end{array}$ \\
\hline Death of the parents & $7.41 \%$ \\
\hline Missing parents & $1.96 \%$ \\
\hline Parents without parental rights & $0.85 \%$ \\
\hline Poverty & $44.27 \%$ \\
\hline Abuse and neglect & $21.69 \%$ \\
\hline Disability of child & $10.79 \%$ \\
\hline Disability of parent & $3.89 \%$ \\
\hline Other & $9.14 \%$ \\
\hline
\end{tabular}

Clear definitions and proceedings

In the process of improvement of the monitoring report an identified problem was the lack of differentiated definitions regarding of the gravity of abuse. The implementation proceedings are communicated officially and training sessions for professionals from every county directorate responsible with the implementation of the instrument were held.

Due to administrative realignment the county directorates have the duty of monitoring of domestic violence cases, too. Law 217/2003 for 
preventing and combating family violence operates with the concept of violence, without mentioning abuse and its forms that is used in Law 272/2004. The Penal Code only uses the concept of maltreatment. There is a clear need for harmonizing legal concepts and definitions in the domain of child abuse and family violence. As a result, development of a common database on child abuse and neglect and domestic violence could be possible.

Due to the fact that the national database is not child-centered, many of the socio-demographic, incident and perpetrator related data are missing, it doesn't offer relevant information neither regarding the phenomenon of abuse, nor about the evolution of the child protection system.

Concerning the relevance of the variables, knowing that the present set of variables are not corresponding to the needs of monitoring institution, it is in the process of evaluation and improvement, in piloting phase in few counties, as representative of NAPFCR communicated.

\section{Institutional and legal framework of data collection on local level}

On local - county - level child protection services are provided by county directorates for social assistance and child protection (DGASPC), mainly for children who are separated from their families. On commune and municipal level the responsible institution is the public social service, which offers secondary prevention services for children and families.

Obligation of reporting and sanctions in case of disregarding the reporting duty Law 272/2004 stipulates the obligation of reporting for professionals of any suspicions of abuse (art. 91(1)). There are no sanctions in case of lack of observance of reporting duty.

Identification of responsible institutions on central, regional and local level

Law 272/2004 and secondary legislation specifies the responsible institutions and stipulates the development of an appropriate institutional infrastructure, internal mechanisms and proceedings which to allow the valid and correct registration of abuse cases. 
Article 91(2) foresees the establishment of specialized departments named 'Child's Phone', a publicly known number, which to respond to the abuse reports. The secondary legislation (Order no. 177/2003) foresees the permanent functioning of a mobile team, specialized for intervention in abuse cases. This service has the duty of immediate evaluation of the risk the child is laying under and of undertaking immediate measures of protecting the child, if it is necessary.

The same document provides a reporting monitoring report and a primary evaluation form that are to be used by professionals.

Law 272/2004 stipulates the duty of public social service (on commune and municipal level) to identify abuse cases, and the duty of county directorates to make the primary evaluation for every reported abuse case.

On the other hand, professionals must report the suspicion of abuse to one of the two above mentioned institutions; law is not specifying the case when the one or the other institution should be contacted.

If no clear protocol exists between these institutions, then serious overlaps of actions could result, as one interviewee has mentioned.

The interviews also showed that only the cases reported such as abuse are figuring in abuse statistics. Other cases, registered as 'social cases' could cover also abuse, but they remain unrevealed.

The fact that not every case is evaluated from the point of view of abuse is rooted also in evaluation instruments used by the public social services, which contains no explicit head and screening questions regarding of abuse incidence. It is only one head, named 'problems of family/children' where the social worker can mention an eventual abuse episode. But in a lack of special training regarding abuse and neglect, there is a very little chance that the social worker would recognize the signs and symptoms of a abuse with the occasion of one visit. At the same time, there are no questions regarding the parenting capacities of caretakers, however, this is the document on which rely the public social service's request for protection measure in the favor of the child, submitted to the county directorate. 
Results of the questionnaires regarding the databases monitoring report on county level

Out of 47 questionnaires 43 were filled in and sent back. They were filled in by a representative of the county directorates, from the specialized service for abuse and neglect.

Results regarding institutional system of data registration and collection

Data are requested in every four month, starting from January for the last trimester of the previous year.

Standardized forms for reporting and registration

20 counties $(44.4 \%)$ have standardized forms for reporting and registration of cases.

Protocols which coordinates the different data generating sectors

Only 8 counties $(18.6 \%)$ have declared that there is a common protocol signed by different institutions in the county in order to avoid case duplication.

Guidelines for implementation of definitions and methodologies

In $50 \%$ of the responding counties there is an internal guide or manual which describes the methodology of case registration. In $73.3 \%$ of the counties there is no internal coding system to avoid duplication of registration and parallel work on cases. By those who have a certain system, the following methods were mentioned: coding number (6 counties), personal identification number ( 2 counties), internal registering software (1 county).

\section{Data validity}

Only 12 counties consider that their own data regarding abuse reflects the reality in their county. 29 counties consider that the statistics of child abuse and neglect is used in their county for program planning and implementation. 


\section{Electronic database and user-friendly software for archive}

43 counties declare to have a database regarding child abuse and neglect (see the format of databases in Table no. 2 .

Table 2. Format of data base on county level

\begin{tabular}{|l|l|}
\hline Format of data base on county level & $\begin{array}{l}\text { Number of } \\
\text { counties }\end{array}$ \\
\hline $\begin{array}{l}\text { Data base exists (electronic and/or paper supported) only at } \\
\text { theMonitoring Department }\end{array}$ & 10 \\
\hline $\begin{array}{l}\text { Electronic data base exists on child abuse and neglect at the Abuse } \\
\text { Department and another data base exists at the Monitoring Department }\end{array}$ & 26 \\
\hline $\begin{array}{l}\text { Electronic data base exists at the Monitoring Department, and in Abuse } \\
\text { Department exists a paper supported register }\end{array}$ & 6 \\
\hline $\begin{array}{l}\text { There is no uniform data base at the Monitoring Department, but every } \\
\text { department have it's own register of cases }\end{array}$ & 1 \\
\hline $\begin{array}{l}\text { The electronic data base from the Monitoring Department has special } \\
\text { items regarding child abuse and neglect }\end{array}$ & 1 \\
\hline
\end{tabular}

Results regarding existing information in county level database - descriptive variables regarding situation, measures and context

The questionnaire refers only to information that exists in formal databases, without information which could exist in personal files of the children. More information is monitoring report related to the victim (see Table 3 ).

Table 3. Available data about victim

\begin{tabular}{|l|l|}
\hline Disposable data about victim & \% counties \\
\hline Date of birth & 88.1 \\
\hline Age & 97.6 \\
\hline Sex & 97.6 \\
\hline Education & 67.4 \\
\hline
\end{tabular}


There is a lack of information regarding the characteristics of abuse incident (see Table 4), and family situation (see Table 5) and perpetrator (see Table 6).

Table 4. Available data about abuse incidents

\begin{tabular}{|l|l|}
\hline Disposable dat a about abuse incident & \% counties \\
\hline Type of abuse & 100 \\
\hline Description of incident & 59,5 \\
\hline Duration & 45,2 \\
\hline Date when it started & 38,1 \\
\hline Number of incidents & 54,8 \\
\hline Gravity & 54,8 \\
\hline Reporting person & 82,9 \\
\hline Number of reports & 72,5 \\
\hline Short term effects & 47,6 \\
\hline Long term effects & 35,7 \\
\hline Protection measures for the child & 97,6 \\
\hline Services provided for the victim & 83,3 \\
\hline
\end{tabular}

Table 5. Available data about family

\begin{tabular}{|l|l|}
\hline Disposable data about abuse family & \% counties \\
\hline Family satus & 69,0 \\
\hline Family income & 47,6 \\
\hline Co-habitants & 52,4 \\
\hline $\begin{array}{l}\text { Information about violence against one parent } \\
\text { or other adult member of the family }\end{array}$ & 57,1 \\
\hline $\begin{array}{l}\text { Information about incidents of abuse or neglect } \\
\text { against other minor members of the family }\end{array}$ & 54,8 \\
\hline
\end{tabular}

Table 6. Available data about perpetrator

\begin{tabular}{|l|l|}
\hline Disposable data about perpetrator & \% counties \\
\hline Sex & 69,0 \\
\hline Age & 52,4 \\
\hline $\begin{array}{l}\text { Relationship between child victim and } \\
\text { perpetrator }\end{array}$ & 73,8 \\
\hline Measures taken against perpetrator & 57,1 \\
\hline
\end{tabular}




\section{Discussion}

First of all we can state that the national database which relies on the Quarterly monitoring report is not case-centered, but it is the only one which has specific information about child abuse and neglect in Romania. The institutional and legal framework constitutes an important starting point for developing a national case-based surveillance system.

The actual database is missing important variables recommended by relevant studies in order to get a clearer image of the phenomenon of child abuse and neglect. Information is missing regarding the socio-economical status of the child victim (we have so far only sex, age and type of residence) and of the perpetrator. Nevertheless, we have more information on actions taken (decision of separation of the child of the family) and type of services provided. We don't have any information about services provided to other family members. But, due to the fact that the database is not case-centered, we can not know, what is the percentage of children have benefited from and what kind of services, if there is a correspondence between type of abuse and services provided etc. In one word, no analysis is possible, beyond the rough numbers of registered cases, which also does not reveal trends or evolutions and efficiency of the system, only the development of reporting capacity of institutions.

If we look to the reported cases since 2007 we can see that the number of identified cases is relatively constant (see official statistics published by the Ministry of Labor, Family and Social Protection). On the other hand, we can notice a great deal of doubt regarding the accuracy of data on central level as a consequence of existing gaps in reporting mechanism. In the same time there is a lack of confidence regarding collected data on local (county) level, the most evoked reason is that professionals from commune and municipal level don't report abuse cases.

The issue of accurate evaluation was raised both by county directorates and public social services: there is no standard evaluation proceeding applied to children, beneficiaries of protection measures in order to evaluate the trauma suffered. This could be the reason why there is only a small percentage of children separated from their families because of abuse and neglect. The instruments used do not contain screening items. This way, cases are registered such as abuse cases only if are reported as such. Thus, 
the extra role of social services of identifying abuse cases among service users who do not request support for abuse, can not be fulfilled. In the circumstances in which social workers are not trained (which is the case in Romania, according to official data) and do not have special instruments, the chance to identify maltreatment at a so-called social case is very small.

There are legislative inadequacies regarding the definitions of abuse and responsible institutions for initial evaluation. Consequently many cases are evaluated by both institutions which were noticed, having negative impact both on clients' lives and effective management of resources.

The chapter Costs is not included in the present analysis, because in 2009 has been introduced the standard cost per beneficiary of protection and prevention services. The implementation of the standard is worth to be analyzed after the first year. On the other hand there is no estimation of the total costs involved by interventions made by different other institutions beside social services in abuse and neglect cases.

\section{Conclusions}

\section{Opportunities for improvement of national data system on child abuse and neglect in Romania}

On legislative level there is a need of harmonization of Civil Code and Penal Code regarding definitions of child abuse and neglect, family violence and maltreatment and correlating with the monitoring instrument. Harmonization of the special law and secondary legislation is also needed, regarding the institutional responsibility of initial evaluation of abuse and neglect. It is also important to analyze the possibility to introduce sanctions in case of disregarding to report maltreatment cases.

Improvement of the variables included in the monitoring report has a great importance, in order to remark trends of the phenomenon. This is possible only if the appropriate indicators are used. On institutional level it is important to integrate data bases used by different departments (usually the monitoring department and abuse department), to have an easier reporting mechanism on the one hand, on the other hand this has a great significance in case management and monitoring. 
For an administrative control, efficient planning and evaluation of the system's development, variables related to the personnel and finances must be included in the monitoring tool.

It is necessary to develop standard and unified working instruments on national level to facilitate the screening of abuse with the occasion of providing any type of social services. These instruments also should ensure the correct evaluation and registration of cases, which leads to even more evidence based decision making regarding the issue of the separation of the child from his family. These instruments must be supported by appropriate guidelines and manuals in order to make sure that the instruments are applied as uniformly as possible nationwide.

Correlation of definitions used in different sectors (social, justice, health, education), including common items in databases of each sector and signing protocols between them regarding the issue of data collection and correlation on local (county) and central level is necessary to get a real picture of the phenomenon, that could show a real interest on the part of the state regarding the assumed responsibility of protecting children from violence of any kind.

The continuous training of personnel involved in data collection that considers the personnel fluctuation, and awareness rising among policy makers on the issue of valid data, needs more human and financial resources.

\section{Acknowledgements}

This work was supported by the Balkan Epidemiological Study on Child Abuse and Neglect, initiated by Institute of Child Health, Department of Mental health and Social Welfare, Centre for the Study and Prevention of Child Abuse and NeglectGreece and run under the auspices of the European Commission and co-funded by DG Research in the context of FP7-Cooperation.

\section{References:}

Alexandrescu, Gabriela. 2000. Child abuse and neglect. Report. Save the Children Romania.

Creighton, Susan J. and Neil Russell. 1995. Voices of Childhood. London: NSPCC. 
Doueck, Howard J., Diana J. English, Diane Depanfilis and Gerald T. Moote. 1993. Decision-Making in Child Protective Services: A comparison of selected risk-assessment systems. Child Welfare 72 (5): 441-452.

European Parliament. Decision No 803/2004/EC of the European Parliament and of the Council of 21 April 2004 adopting a programme of community action.

http:/ / eur-lex.europa.eu/LexUriServ / LexUriServ.do?uri=CELEX: 32004D0803:EN:HTML

European Network of National Observatories on Childhood. 2007. Review of national systems of statistics and registration on child abuse.

http://www.childoneurope.org/issues/childabuse/Child-abusegen2007.pdf

Finkelhor, David and Lisa Jones. 2006. Why Have Child Maltreatment and Child Victimization Declined. Journal of Social Issues 62 (4): 685-716.

Fluke, John D. and Lil Tonmyr. 2009. Frameworks for International Comparison of Child Maltreatment Data. ISPCAN.

http://www.ispcan.org/resource/resmgr/world_perspectives/worl d_persp_2008_-_final.pdf

Gough, David and Margaret A. Lynch. 2000. Prevalence, Identification and Assessment. Child Abuse Review 9 (5): 305-309.

Jones, Lisa M., David Finkelhore and Kathy Kopiec. 2001. Why is sexual abuse declining? A survey of state child protection administrators. Child Abuse and Neglect 25 (9): 1139-1158.

Ministry of Labor, Family and Social Protection. Protecția copilului. http:/ / www.copii.ro/statistici.html?id=96

Palmer, Sally E., Ralph A. Brown, Naomi I. Rae-Grant and Joanne M. Loughlin. 1999. Responding to children's disclosure of familial abuse: what survivors tell us. Child Welfare 78 (2): 259-282.

Reading, Richard, Susan Bissell, Jeffrey Goldhagen, Judith Harwin, Judith Masson, Sian Moynihan, Nigel Parton, Marta Santos Pais, June Thoburn and Elspeth Webb. 2009. Promotion of children's rights and prevention of child maltreatment. The Lancet.

http:/ / www.thelancet.it/journals/lancet/article/PIIS0140-6736(08) 61709-2/fulltext (accessed May 1, 2011)

World Health Organization. 2002. The world health report 2002 - Reducing Risks, Promoting Healthy Life. http://www.who.int/whr/2002/en/

United Nations Committee on the Rights of the Child. 2009. Concluding Observation ROMANIA http:/ / www.unicef.org/romania/CRC-C-ROM-CO-4.pdf

United Nations. 2002. World Fit for Children http://www.unicef.org/specialsession/docs_new/documents/ARES-S27-2E.pdf 